\title{
Raquitismo resistente hipocalcémico, forma pseudocarencial o de Prader
}

Dres. Francisco Beas, "Marta Eggers, ${ }^{*}$ Eduardo Weich"*.

El raquitismo resistente hipocalcémico es una forma especial de raquitismo caracterizada por comienzo precoz (se manifiesta antes del año de edad); se acompaña: de hipocalcemia difícil de corregir, que frecuentemente se traduce por tetania; de hipofosfemia moderada, corregible con el tratamiento; de aminoaciduria generalizada y de hipercloremia. Tiene herencia autosómica dominante y por lo general no responde a las dosis habituales de vitamina $D$. A esto se agregaría, posteriormente, defecto del esmalte de los dientes definitivos.

Esta forma especial de raquitismo resistente fue descrita por Prader en 1961, quien recopiló varios casos de la literatura y comunicó otros propios, y estableció las diferencias que lo distinguen del raquitismo resistente hipofosfémico familiar. Lo denominó Raquitismo Resistente Familiar Pseudocarencial por su parecido clínico y de laboratorio con el raquitismo carencial simple, del que se diferencia por la falta de respuesta a las dosis habituales de vitamina $\mathbf{D}$ y en la presentación familiar.

Sería una afección muy poco frecuente, existiendo pocos casos comunicados, y de éstos los hay muy acentuados y otros más leves, siendo los requerimientos de vit. D muy variables.

Su etiopatogenia no está aún bien establecida, pero probablemente se deba a un trastorno en la metabolización de la vit. $\mathrm{D}$, a nivel de su conversión a metabolitos activos. Esta falla podría ser de grado variable, lo que explicaría que algunos pacientes requieran dosis menores de vit. $\mathrm{D}, \mathrm{y}$ otros casos, dosis altísimas. La absorción intestinal de la vit. $\mathrm{D}$, cuando ha sido estudiada en este tipo de paciente, se ha encontrado normal.

\footnotetext{
- Unidad Endrocrino genética, Hospital Arriarán.

* Servicio de Medicina, Hospital Arriarán.
}

A continuación se presenta un caso estudiado en el Servicio de Endocrinología del Hospital Arriarán, que por sus características clínicas, de laboratorio, tipo de respuesta al tratamiento y antecedente familiar positivo, estimamos que corresponde a esta forma pseudocarencial descrita por Prader.

\section{CASO CLINICO}

I.E.M., dos años de edad, referido para estudio por presentar un raquitismo florido, a pesar de haber recibido vit. $\mathrm{D}^{2} 600.000$, en forma repetida desde el mes de edad.

Antecedentes familiares. Padre y madre jóvenes, sanos, no parientes entre sí. Sin deformaciones óseas, de estatura normal. La madre ha tenido sólo dos embarazos. primero una niña que falleció a los 11 meses de bronconeumonía y que habría presentado deformaciones torácicas atribuidas a raquitismo, pero que no alcanzó a ser estudiada. El segundo hijo es el paciente presentado. No hay antecedentes de otros familiares con talla baja o deformaciones óseas.

Antecedentes personales Embarazo normal. Primeros movimientos fetales al cuarto mes. Parto de término, normal. Peso de nacimiento 3.820 gramos. Período RN, normal.

Alimentactón. Lactancia, 1 mes. Luego leche y harinas en cantidad y concentración adecuadas, indicados en los controles médicos. Comida desde el tercer mes. Huevo, carne y legumbres desde el sexto mes. Rechazo parcial de la alimentación desde los ocho meses.

Desarrollo psicomotor. Aparentemente normal hasta el año; sonrie a los dos meses, se sient= a los seis meses, dentición a los 3 meses, camina con apoyo desde los 10 meses, primeras palabras alrededor del año. Nunca caminó solo, apreciándose deterioro del desarrollo motor después del 1 1/2 año, llegando a ser incapaz de sentarse solo a los dos años. No controla esfinteres.

Enfermedades anteriores. Bronquitis a repetición. Diarrea de corta duración, un episodio

Enfermetad actual. Habría sido eutrófico hasta aproximadamente los nueve meses de edad, presentando sólo resfríos y bronquitis. Su control médico periódico 
lo efectuaba en forma particular. A los nueve meses el médico tratante hace el diagnóstico de raquitismo, consignado la presencia de cráneo tabes +++ . Hasta ese momento habia recibido vit. $\mathrm{D}_{2} 600.000 \mathrm{C}$, dos veces, oral, al mes y a los tres meses. El médico indica como tratamiento del raquitismo vit. $\mathrm{D}_{2}$ oral $600.000 \mathrm{U}$, cada 15 días por tres veces, lo cual la madre aseguró haber cumplido. Posteriormente, al año de edad, la madre nota deformación gra. dual del tórax, similar a la presentada por la hermana fallecida.

Es controlado al año tres meses constatándose persistencia de cráneo tabes y tórax en quilla, por lo que se indica repetir 3 veces más vit. $D_{2} 600.000 \mathrm{U}$. oral cada 15 dias.

En controles posteriores el médico indica nuevos golpes vitamínicos cada 20 días, tratamiento que la madre asegura haber cumplido. Posterirmente aparecen dolores en las extremidades, cansancio fácil, sudoración importante. La talla y el peso se estacionan comprometiéndose progresivamente el estado general, además se hacen notorias, deformaciones en las extremidades superiores $e$ inferiores, con dolor importante a la movilización, por todo lo cual es referido para su estudio.

Examen físico. Niño de dos años en malas condiciones generales y nutritivas. Habla y coopera al examen. Sudoración profusa, hipotonía muscular franca. Talla 74 cm. Peso, 8.500 gramos, circunferencia craneana, $49 \mathrm{~cm}$ Presión arterial 100/65. Respiraciones, $30^{+}$. Pulsa, $140 \mathrm{X}$.

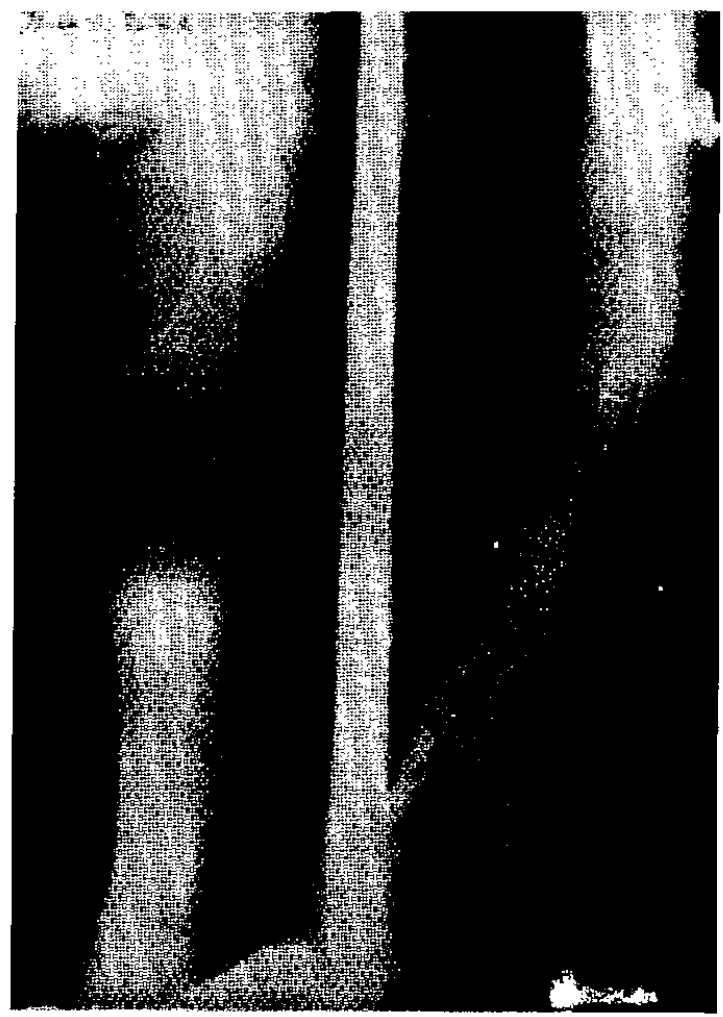

Fig. 1

Radiografía de huesos largos, metáfisis de tipo raquitico, previo al tratamiento.

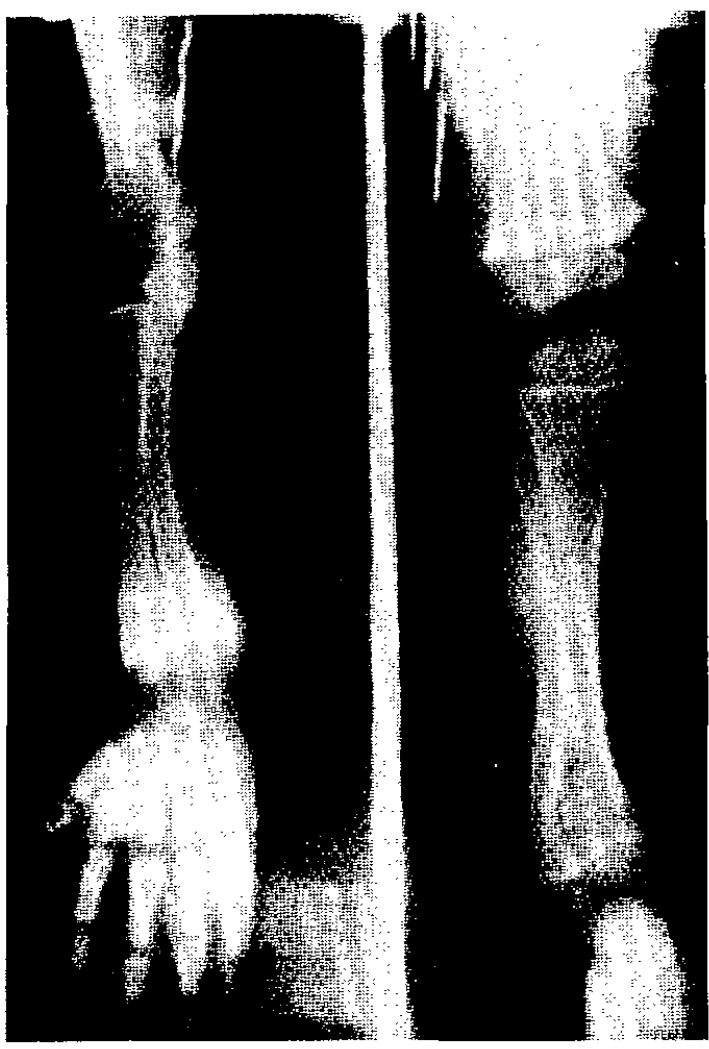

Fig. 2

Radiografía de huesos largos, a los 3 meses de tratamionto. Signos de reparación parcial.

Bregma abierto $1,5+1 \mathrm{~cm}$, normotenso. Cráneo tabes ${ }^{+}$

Hipertelorismo moderado (de tipo familiar). Escleras algo azulejas. Dientes en buenas condiciones:

$$
\frac{11411}{11411}
$$

Cuello: nada especial.

Columna: xifosis dorsal baja

Fórax: deformación en quilla, rosario costal ${ }^{+}$, pulmón: roncus y estertores medianos difusos regular cantidad.

Corazón: tonos bien timbrados, taquicardia.

Abdomen: depresible, algo glubuloso. Hígado y bazo dentro de los límites normales. No se palpan riñones.

Genitales: masculinos, normales

Extremidades: engrosamiento epifisiario generalizado, deformación de ambas tibias con incurvación anterior. Se plantean los diagnósticos de raquitismo resistente y desnutrición, siendo hospitalizado para su estudio, solicitándose exámenes de tipo general y especial, orientados a pesquisar alteración renal, intestinal o metabólica que pudiera relacionarse con su raquitismo. Estos exámenes están resumidos en la tabla 1

El estudio radiológico reveló rarefacción del esqueleto; metafisis de tipo raquítico; fracturas a nivel del tercio medio del fémur y tercio distal del radio. Pequeñas fracturas a nivel de los metacarpianos (Figs. 1 y 2). Pielogragía normal 
Tabla 1

RESUMEN DE EXAMENES DE LABORATORIO, MADRE, PADRE, PACIENTE

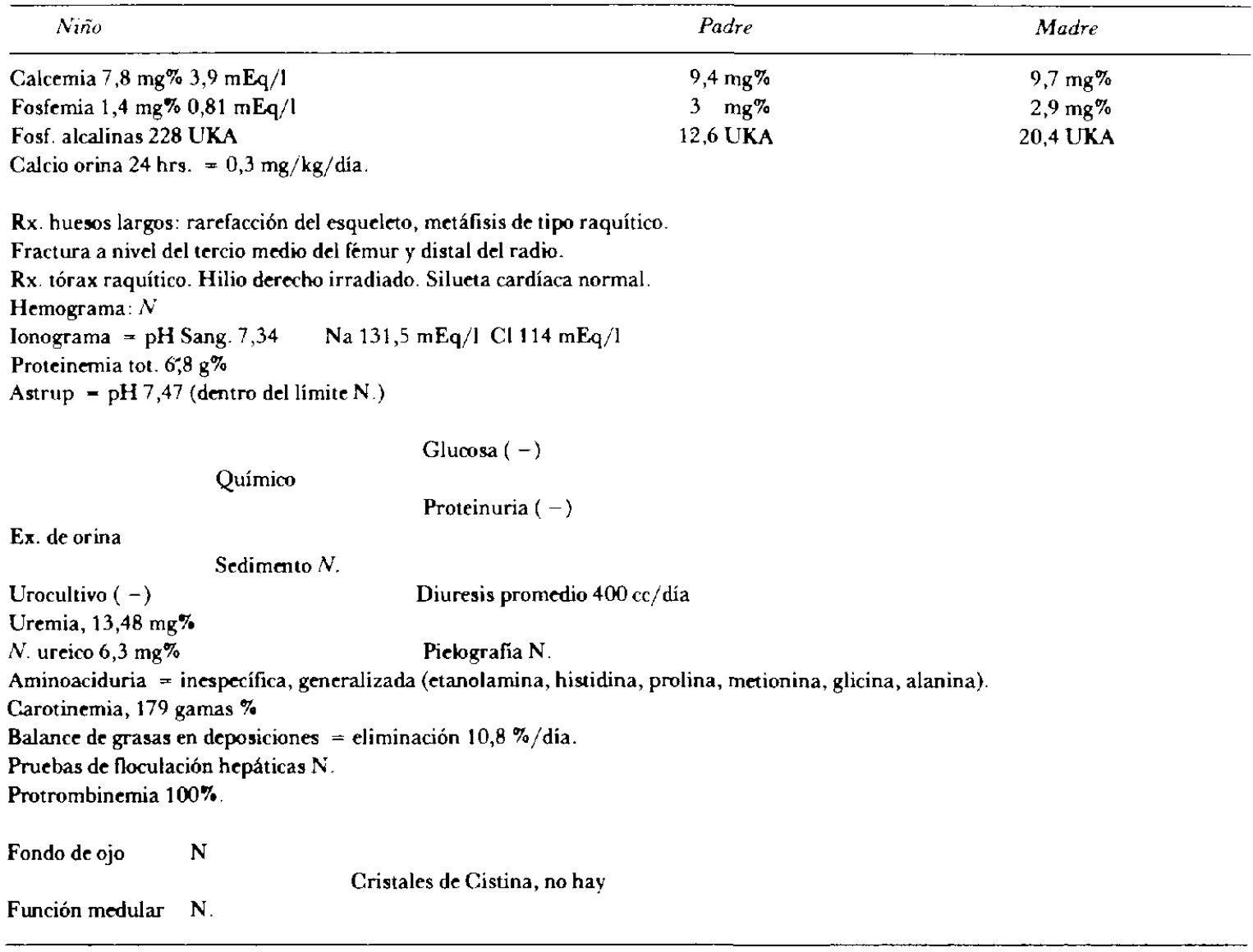

De acuerdo con estos resultados iniciales, con fosfemia baja, calcemia algo baja y fosfatasas alcalinas muy elevadas, se pensó inicialmente que se trataba de un raquitismo resistente de tipo hipofosfémico, que es la forma más frecuente de observar.

\section{EVOLUGION, TRATAMIENTO Y DISCUSION}

Se inició tratamiento con vitamina $\mathrm{D}_{2}$ $600.000 \mathrm{U}$ oral y solución de fosfatos, $15 \mathrm{ml}$ 3 veces al día, lo que aporta $+-1 \mathrm{~g}$ de $P$. al día. Previo control de calciuria, calcemia y fosfemia, se fue repitiendo la vit. $\mathrm{D}_{2} 600.000 \mathrm{U}$ oral, inicialmente a los 10 días y luego cada 2 días, además de solución de fosfatos.

Después de las primeras semanas de tratamiento, llamó la atención una respuesta no esperada, que no se ve al tratar un raquitismo resistente de tipo hipofosfémico. Se produjo una rápida normalización de la fosfemia, mientras que la calcemia se hizo francamente baja. Al mes de tratamiento, después de haber recibido 5 golpes vitamínicos, persistía hipocalcemia importante de $5,7 \mathrm{mg} \%$, mientras que la fosfemia había subido a 5,6 mg\% (Fig. 3).

Este tipo de respuesta no se ve nunca al tratar un raquitismo hipofosfémico, ya que en éstos sólo se logra corregir parcialmente la fosfemia, sin llegar a valores normales y, por lo demás, no se acompañan de hipocalcemia.

Esto orientó a pesar en la forma pseudocarencial descrita por Prader, ya que concordaban las siguientes características:

- Comienzo precoz: antes del año de edad.

- Antecedente familiar: una hermana afectada.

- Hipocalcemia: a veces con tetania, que se demora en corregir con tratamiento. 


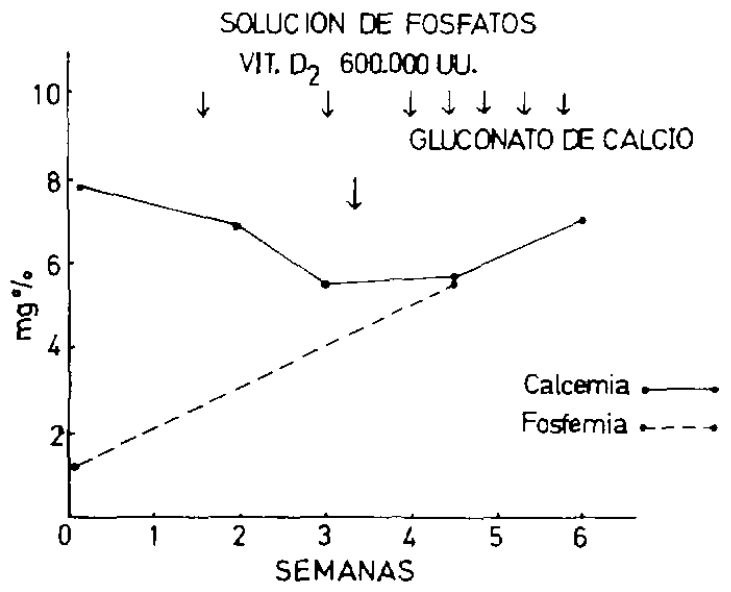

Fig. 3

Respuesta inicial al tratamiento en raquitismo resistente.

- Hipofosfemia: que se corrige con tratamiento.

- Aminoaciduria generalizada.

- Ionograma con cloro aumentado.

Se continuó tratando con vit. $\mathrm{D}_{2} 600.000$ U oral cada 2 días, solución de fosfatos y gluconato de $\mathrm{Ca}$, apreciándose a los 2 meses de tratamiento la aparición de signos radiológicos de reparación. Las alteraciones bioquimicas tardaron más tiempo en corregirse.

Fue dado de alta después de 3 1/2 meses de tratamiento, apreciándose una franca recuperación clínica, radiológica y bioquímica, persistiendo sólo un moderado aumento de las fosfatasas alcalinas.

La evolución posterior ha sido muy satisfactoria, requiriendo como tratamiento de man- tención vit. $\mathrm{D}_{2} 600.000 \mathrm{U}$ orales una vez a la semana, gluconato de calcio $3 \mathrm{gr}$ al día y solución de fosfato $15 \mathrm{ml}$ dos veces al día.

En cada control se efectúa calcemia, fosfemia, fosfatasas alcalinas y calciuria de 24 horas, lo que sirve de guía para modificar el tratamiento en caso necesario.

El criterio usado es mantener calciuria diaria que no sobrepase los $8 \mathrm{mg}$ por kilo de peso al dia para evitar riesgo de nefrocalcinosis o litiasis renal.

Se trata de un niño que a pesar de recibir varios golpes de vit. $\mathrm{D}_{2}$ hace un raquitismo intenso con hipocalcemia importante. No se encontró ninguna otra patología asociada luego de haberse efectuado estudio renal, hepático, de absorción intestinal y metabólico.

El diagnóstico de raquitismo resistente hipofosfémico que es la forma más frecuente de raquitismo resistente, no concordaba, ya que en éste las deformaciones raquíticas se presentan después del año de edad, cursa con normocalcemia y la hipofosfemia sólo se logra corregir en forma parcial. Las diferencias entre estos dos tipos están consignadas en tablas 2 y 3 .

Se buscaron otras causas poco frecuentes de raquitismo resistente, descartándose cistinosis, tirosinosis, síndrome de Toni Debré Fanconi y acidosis tubular. Nos parece que este caso concuerda con la forma de raquitismo pseudocarencial descrita por Prader, por su similitud clinica con el iraquitismo carencial simple: comienzo precoz, rosario costal, cráneo tabes, hipocalcemia, aminoaciduria gene-

Tabla 2

TIPOS DE RAQUITISMO. CUADRO COMPARATIVO

Raquitismo resistente hipofosfémico

Iniciación tardía

Hipotonía muscular ausente

Desarrollo neuromotor normal

Ausencia de tetania

Hipofosfemia ( $<3 \mathrm{M} / \mathrm{g} / 100 \mathrm{Ml}$ )

Calcemia normal

Fosfatasas elevadas

Ausencia de aminoaciduria

Ausencia de acidosis

Persistencia de hipofosfemia con vit.

Reraso de talla a pesar de vit. D

Herencia dominante ligada a cromosoma $X$ o esporádico
Raquitismo pseudocatencial

Iniciación precoz

Hipotonía muscular presente

Retraso desarrollo neuromotor

Tetania frecuente

Fosfernia normal o poco $\downarrow$

Hipocalcemia $(<8 \mathrm{Mlg} / 100 \mathrm{Ml}$ )

Fosfatasas elevadas

Aminoaciduria

Acidosis hiperclorémica ${ }^{+}$

Corrección con vit. D

Aceleración del crecimiento con vit. D

Herencia dominante autosómica 
Tabla 3

TIPOS DE RAQUISMO. CUADRO COMPARATIVO

\begin{tabular}{|c|c|c|c|}
\hline & Raquitusmo resistente & Raquitismo resistente & Raquitismo carencial \\
\hline & Hipofosfémico & Hipocalcémico & \\
\hline Comienzo & >> 1 aก̃o & $<1$ año & $<1$ an̄o \\
\hline Tetania & No & Frecuente & Frecuente \\
\hline Calcemia & $\mathrm{N}$ & † Dificil de corregir & $N$. o ligeramente $\downarrow$ \\
\hline Fosfemia & t Dificil de corregir & $\pm \downarrow$ Fácil de corregir & $\downarrow$ Fácil de corregir \\
\hline Fosfatasas alcalinas & $\uparrow$ & $\uparrow$ & $\uparrow$ \\
\hline Aa. orina & Poco frecuente & Frecuente & Frecuente \\
\hline \multicolumn{4}{|l|}{ Alteraciones esmaite } \\
\hline $2^{a}$ dentición & No & Frecuente & Frecuente \\
\hline Herencia & $\begin{array}{c}\text { Dominante ligada a } \\
\text { cromosoma } X\end{array}$ & Autosómica dom. & -- \\
\hline
\end{tabular}

ralizada, diferenciándose solamente en su falta de respuesta a las dosis habituales de vitamina D.

\section{RESUMEN}

Se describen las caracteristicas del raquitismo resistente hipocalcémico descrito por Prader.

Se presenta el caso de un niño tratado por raquitismo desde los nueve meses de edad, con golpes vitamínicos múltiples, sin respuesta a este tratamiento, acentuándose el cuadro clínico. El estudio clínico, bioquímico y radiológico resultó similar al de un raquitismo carencial intenso, diferenciándose en la falta de respuesta al tratamiento habitual. Requirió dosis altísimas de vit. D asociada a solución de fosfatos y calcio oral. Se descartan otras causas de raquitismo resistente. Se destaca el antecedente de una hermana afectada. que concuerda con la herencia autosómica dominante descrita en estos casos.

\section{SUMMARY}

A case with the clinical and biochemical features of the Hypocalcemic Resistant Ricket comunicated by Prader is described.

The case is a child who was treated with multiple doses of vit. D for a rickets, diagnosed at 9 months of age, with no response and progressive involvement, requiring hospitalization at age 2 years. The clinical, biochemical and radiological study was similar to vit. D deficiency rickets, differing by the no response to usual treatment. He required very high doses of vit. D associated with phosphate solution and oral calcium.

There were no other pathological findings and other causes of resistant rickets were not found.

\section{REFERENCIAS}

Jonxis, J.H.P. Some investigations on rickets. J. Pediat. 59: 607,1961 .

Lytt I. Gardner. Endocrine and Genetic Diseases of Childhood, 1975.

Prader, A. Ruth Illig und Emmi Heierla. Eine besondere Form, der primaren vitamin -D- resistent Rachitis mit Hypocalcamie und autosomal-dominantemi Erbgang: die hereditare Pseudo - Mangel rachitis. Helvetica Paediatrica Acta, Vol. 16, Fasc. 5/5, XII, 1961

Rasmussen H., H. de Luca, C. Amaud, C. Hawket, M. von Stedingk. The relationship between $V i t . \mathrm{D}$ and parathyroid hormone, J. Clin. Invest. 42:1940, 1963.

Sonano, J.R., A. Einhom. Starkh. Edelmann C.R. Deficiency - Type rickets due to decreased sensitivity to Vit. D. J. Pediat. Vol. 68, N².11/66.

Trabajo entregado para su publicación en 1976. 